\title{
On Channels with Action-Dependent States
}

\author{
Behzad Ahmadi and Osvaldo Simeone
}

\begin{abstract}
Action-dependent channels model scenarios in which transmission takes place in two successive phases. In the first phase, the encoder selects an "action" sequence, with the twofold aim of conveying information to the receiver and of affecting in a desired way the state of the channel to be used in the second phase. In the second phase, communication takes place in the presence the mentioned actiondependent state. In this work, two extensions of the original action-dependent channel are studied. In the first, the decoder is interested in estimating not only the message, but also the state sequence within an average per-letter distortion. Under the constraint of common reconstruction (i.e., the decoder's estimate of the state must be recoverable also at the encoder) and assuming non-causal state knowledge at the encoder in the second phase, we obtain a single-letter characterization of the achievable rate-distortioncost trade-off. In the second extension, we study an action-dependent degraded broadcast channel. Under the assumption that the encoder knows the state sequence causally in the second phase, the capacitycost region is identified. Various examples, including Gaussian channels and a model with a "probing" encoder, are also provided to show the advantage of a proper joint design of the two communication phases.
\end{abstract}

Index Terms

Action-dependent channels, state amplification, degraded broadcast channels, common reconstruction constraint.

\section{INTRODUCTION}

In [1], the framework of action-dependent channels was introduced as a means to model scenarios in which transmission takes place in two successive phases. In the first phase, the encoder selects an "action" sequence, with the twofold aim of conveying information to the

B. Ahmadi and O. Simeone are with the CWCSPR, New Jersey Institute of Technology, Newark, NJ 07102 USA (e-mail: \{behzad.ahmadi,osvaldo.simeone\}@njit.edu). 
receiver and of affecting in a desired way the state of the channel to be used in the second phase. In the second phase, communication takes place in the presence the mentioned actiondependent state. With a cost constraint on the actions in the first phase and on the channel input in the second phase, reference [1] derived the capacity-cost-trade-off under the assumption that the channel state is available either causally or non-causally at the encoder in the second phase.

A number of applications and extensions of the results in [1] have been reported since then. In [2], the result in [1] is leveraged to study a model in which encoder and decoder can "probe" the channel state to obtain partial state information during the first communication phase. In [3], unlike [1] the decoder is required to decode both the transmitted message and channel input reliably. Finally, in [4], the decoder is interested in estimating not only the message but also the state sequence, and the latter is available strictly causally at the encoder in the second transmission phase.

In this paper, two further extensions of the original action-dependent channel are studied. In the first, similar to [4], the decoder is interested in estimating not only the message but also the state sequence within given average per-letter distortion constraints (see Fig. 1). Unlike [4], we assume non-causal state knowledge in the second phase, and, under the constraint of common reconstruction (CR) (i.e., the decoder's estimate of the state must be recoverable also at the encoder with high probability [5]), we obtain a single-letter characterization of the achievable rate-distortion-cost trade-off. We remark that, for conventional state-dependent states without actions, the problem of joint estimation of message and state with non-causal state information at the encoder without the CR constraint is open (see, e.g., [6]), while with the CR constraint the problem has been solved in [5]. In the second extension, illustrated in Fig. 2, we study an action-dependent degraded broadcast channel. Under the assumption that the encoder knows the state sequence causally in the second phase, the capacity-cost region is identified 1$]$ The corresponding result for action-independent states was derived in [9] (see also [10]), while we recall that with non-causal state information the problem is open (see [11]). Various examples, including Gaussian channels and a model with a "probing" encoder, are also provided throughout to show the advantage of a proper joint design of the two communication phases.

\footnotetext{
${ }^{1}$ After submitting [7], we have been informed of the reference [8], where the problem illustrated in Fig. 2 has also been solved.
} 


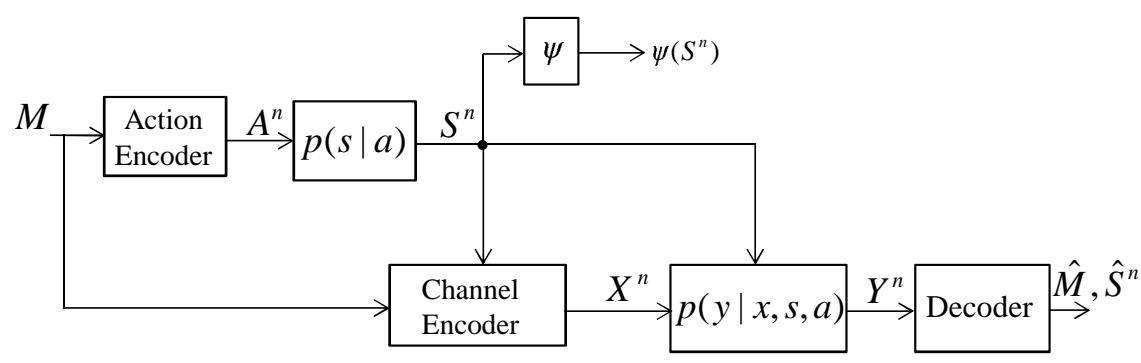

Figure 1. Channel with action-dependent state in which the decoder estimates both message and state, and there is a common reconstruction $(\mathrm{CR})$ constraint on the state reconstruction. The state is known non-causally at the channel encoder.

\section{Transmission of Data and Action-Dependent State with Common RECONSTRUCTION CONSTRAINT}

In this section, we study the setting illustrated in Fig.1 of a channel with action-dependent state in which the decoder estimates both message and state. We first detail the system model in Sec. III-A. Next, the characterization of the trade-off between the achievable data rate and state reconstruction distortion is derived in Sec. II-B, Finally, a Gaussian example is given in Sec. II-C.

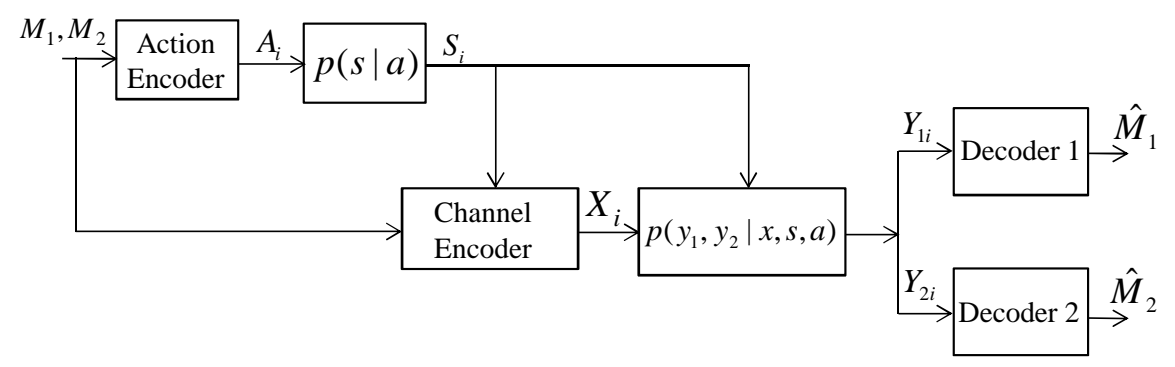

Figure 2. Broadcast channel with action-dependent states known causally to the encoder (i.e., the $i$ th transmitted symbol $X_{i}$ is a function of messages $M_{1}, M_{2}$ and the state symbols up to time $i, S^{i}$ ).

\section{A. System Model}

In this section the system model is detailed. The system is defined by the probability mass functions (pmfs) $p(x), p(y \mid x, s, a), p(s \mid a)$ and discrete alphabets $\mathcal{X}, \mathcal{A}, \mathcal{S}, \hat{\mathcal{S}}$, and $\mathcal{Y}$ as follows. Given the message $M$, selected randomly from the set $\mathcal{M}=\left[1,2^{n R}\right]$, an action sequence $A^{n} \in \mathcal{A}^{n}$ is selected. As a result of this selection, the state sequence $S^{n} \in \mathcal{S}^{n}$ is generated as the output of 
a memoryless channel $p(s \mid a)$ so that we have $p\left(s^{n} \mid a^{n}\right)=\prod_{i=1}^{n} p\left(s_{i} \mid a_{i}\right)$ for an action sequence $A^{n}=a^{n}$. The input sequence $X^{n} \in \mathcal{X}^{n}$ is selected based on both message $M$ and state sequence $S^{n}$. The action sequence $A^{n}$ and the input $X^{n}$ have to satisfy an average cost constraint defined by a function $\gamma: \mathcal{A} \times \mathcal{X} \rightarrow[0, \infty)$, so that the cost for the input sequences $a^{n}$ and $x^{n}$ is given by $\gamma\left(a^{n}, x^{n}\right)=\frac{1}{n} \sum_{i=1}^{n} \gamma\left(a_{i}, x_{i}\right)$. Given $X^{n}=x^{n}, S^{n}=s^{n}$ and $A^{n}=a^{n}$, the received signal is distributed as $p\left(y^{n} \mid x^{n}, s^{n}, a^{n}\right)=\prod_{i=1}^{n} p\left(y_{i} \mid x_{i}, s_{i}, a_{i}\right)$. The decoder, based on the received signal $Y^{n}$, estimates the message $M$ and the sequences $S^{n} \in \mathcal{S}^{n}$. The estimate $\hat{S}^{n} \in \hat{\mathcal{S}}^{n}$ is constrained to satisfy a distortion criterion defined by a per-symbol distortion metric $d(s, \hat{s}): \mathcal{S} \times \hat{\mathcal{S}} \rightarrow\left[0, D_{\text {max }}\right]$ with $0<D_{\max }<\infty$. Based on the given distortion metric, the overall distortion for the estimated state sequences $\hat{s}^{n}$ is defined as $d^{n}\left(s^{n}, \hat{s}^{n}\right)=\frac{1}{n} \sum_{i=1}^{n} d\left(s_{i}, \hat{s}_{i}\right)$. The reconstructions $\hat{S}^{n}$ is also required to satisfy the $\mathrm{CR}$ constraint, which imposes that the state estimate be also reproducible at the encoder with high probability, as formalized below.

Definition 1. An $(n, R, D, \Gamma, \epsilon)$ code for the model in Fig. 1 1 consists of an action encoder

$$
g_{1}: \mathcal{M} \rightarrow \mathcal{A}^{n}
$$

which maps message $M$ into an action sequence $A^{n}$; a channel encoder

$$
g_{2}: \mathcal{M} \times \mathcal{S}^{n} \rightarrow \mathcal{X}^{n}
$$

which maps message $M$ and the state sequence $S^{n}$ into the sequence $X^{n}$; two decoding functions,

$$
\begin{array}{r}
h_{1}: \mathcal{Y}^{n} \rightarrow \mathcal{M}, \\
\text { and } h_{2}: \mathcal{Y}^{n} \rightarrow \hat{\mathcal{S}}^{n},
\end{array}
$$

which map the sequence $Y_{1}^{n}$ into the estimated message $\hat{M}$ and into the estimated sequence $\hat{S}^{n}$, respectively; and a reconstruction function

$$
\psi: \mathcal{S}^{n} \rightarrow \hat{\mathcal{S}}^{n},
$$

which maps the state sequence into the estimated state sequence at the encoder; such that the probability of error in decoding the message $M$ is small

$$
\operatorname{Pr}[\hat{M} \neq M] \leq \epsilon
$$


the distortion and cost constraints are satisfied, i.e.,

$$
\begin{aligned}
& \frac{1}{n} \sum_{i=1}^{n} \mathrm{E}\left[d\left(S_{i}, h_{2 i}\left(Y^{n}\right)\right)\right] \leq D+\epsilon \\
& \text { and } \frac{1}{n} \sum_{i=1}^{n} \mathrm{E}\left[\gamma\left(A_{i}, X_{i}\right)\right] \leq \Gamma+\epsilon,
\end{aligned}
$$

where $h_{2 i}\left(Y^{n}\right) \in \hat{\mathcal{S}}$ is the $i$ th symbol of the sequence $h_{2}\left(Y^{n}\right)$, and the CR requirement is verified, namely,

$$
\operatorname{Pr}\left[\psi\left(S^{n}\right) \neq h_{2}\left(Y^{n}\right)\right] \leq \epsilon
$$

We note that, given the definition above, the pmf of the random variables $\left(M, A^{n}, S^{n}, X^{n}, Y^{n}\right)$ factorizes as

$$
\begin{aligned}
p\left(m, a^{n}, s^{n}, x^{n}, y^{n}\right)= & \frac{1}{2^{n R}} \delta\left[a^{n}-g_{1}(m)\right]\left\{\prod_{i=1}^{n} p\left(s_{i} \mid a_{i}\right)\right\} \delta\left[x^{n}-g_{2}\left(m, s^{n}\right)\right] \\
& \cdot\left\{\prod_{i=1}^{n} p\left(y_{i} \mid x_{i}, s_{i}, a_{i}\right)\right\},
\end{aligned}
$$

where $\delta[\cdot]$ is the Kronecker delta function (i.e., $\delta[x]=1$ if $x=0$ and $\delta[x]=0$ otherwise) and the arguments of the pmf range in the alphabets of the corresponding random variables.

Given a cost-distortion pair $(D, \Gamma)$, a rate $R$ is said to be achievable if, for any $\epsilon>0$ and sufficiently large $n$, there a exists a $(n, R, D, \Gamma, \epsilon)$ code. We are interested in characterizing the capacity-distortion-cost trade-off function $C(D, \Gamma)=\inf \{R:$ the triple $(R, D, \Gamma)$ is achievable $\}$.

\section{B. Capacity-Distortion-Cost Function}

In this section, a single-letter characterization of the capacity-distortion-cost function is derived.

Proposition 2. The capacity-distortion-cost function for the system in Fig. 1 is given by

$$
C(D, \Gamma)=\max I(U ; Y)-I(U ; S \mid A)
$$

where the mutual informations are evaluated with respect to the joint pmf

$$
p(a, u, s, x, y)=p(a) p(s \mid a) p(u \mid s, a) p(x \mid u, s) p(y \mid x, s, a)
$$


and minimization is done with respect to the pmfs $p(a), p(u \mid s, a)$ and $p(x \mid u, s)$ under the constraint that there exists a deterministic function $\phi: \mathcal{U} \rightarrow \hat{\mathcal{S}}$ such that the inequalities

$$
\begin{aligned}
\mathrm{E}[d(S, \phi(U))] & \leq D \\
\text { and } \mathrm{E}[\gamma(A, X)] & \leq \Gamma
\end{aligned}
$$

are satisfied. Finally, $U$ is an auxiliary random variable whose alphabet cardinality can be bounded as $|\mathcal{U}| \leq|\mathcal{A}||\mathcal{S}||\mathcal{X}|+2$.

Remark 3. If we let $D \geq D_{\max }$, the result above recovers Theorem 1 of [1]. If instead we have $p(s \mid a)=p(s)$ so that the channel is not action-dependent, we recover Theorem 1 in [5].

The proof of achievability follows using the same arguments as in [1] with the difference that here $U$ is also used to estimate the state $S$ via a function $\phi(U)$. The proof of the converse can be found in Appendix A.

\section{A Gaussian Example}

In this section, we consider a continous-alphabet version of the model of Fig. 1 in which the actions and the channel input are subject to the cost constraints $1 / n \sum_{i=1}^{n} \mathrm{E}\left[A^{2}\right] \leq P_{A}$ and $\frac{1}{n} \sum_{i=1}^{n} \mathrm{E}\left[X_{i}^{2}\right] \leq P_{X}$, respectively; the action channel is given by

$$
S=A+W
$$

where $W \sim \mathcal{N}\left(0, \sigma_{W}^{2}\right)$ and the transmission channel is given by

$$
Y=X+S+Z
$$

where $Z \sim \mathcal{N}\left(0, \sigma_{Z}^{2}\right)$ is independent of $W$. We evaluate the rate $I(U ; Y)-I(U ; S \mid A)$ in (11) by assuming the variables $(A, S, U, X, Y)$ to be jointly Gaussian without claiming the optimality of this choice. Specifically, similar to [1, Sec. VI], we choose $A \sim \mathcal{N}\left(0, P_{A}\right)$,

$$
\begin{aligned}
X & =\alpha A+\gamma W+G \\
\text { and } U & =\delta X+A+\beta W,
\end{aligned}
$$

with $G \sim \mathcal{N}\left(0, P_{X}-\left(\alpha^{2} P_{A}+\gamma^{2} \sigma_{W}^{2}\right)\right)$, where we enforce the constraint $P_{X} \geq\left(\alpha^{2} P_{A}+\gamma^{2} \sigma_{W}^{2}\right)$, and the variables $(A, W, G, Z)$ are all independent of each other. We evaluate then the rate $I(U ; Y)-I(U ; S \mid A)$ as in [1], with the difference that we have the additional constraint (7) on 
the state estimate $\hat{S}$. Assuming the quadratic distortion metric $d(s, \hat{s})=(s-\hat{s})^{2}$, we choose $\hat{S}$ to be the MMSE estimate of $S$ given $U$ and $A$.2 This leads to the constraint

$$
D \geq E\left[(S-\hat{S})^{2}\right]=\operatorname{var}(S \mid U, A)=\sigma_{W}^{2}-\frac{(E[W(U-A)])^{2}}{E\left[(U-A)^{2}\right]},
$$

where $E[W(U-A)]=(\delta \gamma+\beta) \sigma_{W}^{2}$ and $E\left[(U-A)^{2}\right]=\delta^{2} P_{G}+(\delta \gamma+\beta)^{2} \sigma_{W}^{2}$. The rate $I(U ; Y)-I(U ; S \mid A)$ optimized over parameters $(\alpha, \beta, \delta, \gamma)$ under the constraint (17) for different values of the distortion $D$ for $P_{A}=P_{X}=\sigma_{W}^{2}=\sigma_{Z}^{2}=1$ in Fig. (3). Moreover for reference, Fig. 3 shows also the rate achievable if distribution (12) is designed to be optimal for message transmission only as in [1, eq. (95)], and the rate achievable, if $A$ is selected to be independent of the message, namely, $\max I(U ; Y \mid A)-I(U ; S \mid A)$, where the mutual information terms are evaluated with respect to the joint Gaussian distribution given above in (16) under the constraint (17). The performance gains attainable by designing the transmission strategy jointly in the two phases and by accounting for the constraint (17) are apparent.

\section{Degraded Broadcast Channels with Action-Dependent States}

In this section, we study the problem illustrated in Fig. 2 of a broadcast channel with actiondependent states known causally to the encoder. We first detail the system model in Sec. III-A, Next, the characterization of the capacity region for physically degraded broadcast channels is given in Sec. III-B. In Sec. ПII-D, we study the special case of a broadcast channel with a probing encoder in the sense of [2].

\section{A. System Model}

In this section the system model is detailed. The system is defined by the pmfs $p(x), p\left(y_{1}, y_{2} \mid x\right.$, $s, a), p(s \mid a)$ and discrete alphabets $\mathcal{X}, \mathcal{A}, \mathcal{S}$, and $\mathcal{Y}$ as follows. Given the messages $M_{1}$ and $M_{2}$, selected randomly from the sets $\mathcal{M}_{1}=\left[1,2^{n R_{1}}\right]$ and $\mathcal{M}_{2}=\left[1,2^{n R_{2}}\right]$, respectively, an action sequence $A^{n} \in \mathcal{A}^{n}$ is selected. As a result of this selection, the state sequence $S^{n} \in \mathcal{S}^{n}$ is generated as in the previous section. The action sequence $A^{n}$ and the input $X^{n}$ have to satisfy the average cost constraint (8). Given the transmitted signal $X^{n}=x^{n}$, the state sequence $S^{n}=s^{n}$, and the action sequence $A^{n}=a^{n}$, the received signals are distributed as $p\left(y_{1}^{n}, y_{2}^{n} \mid x^{n}, s^{n}, a^{n}\right)=$

\footnotetext{
${ }^{2}$ Note that $U$ in the characterization of Proposition 2 can be always redefined to include also $A$ without loss of performance, and hence $\hat{S}$ can be made to be a function of $U$ and $A$.
} 


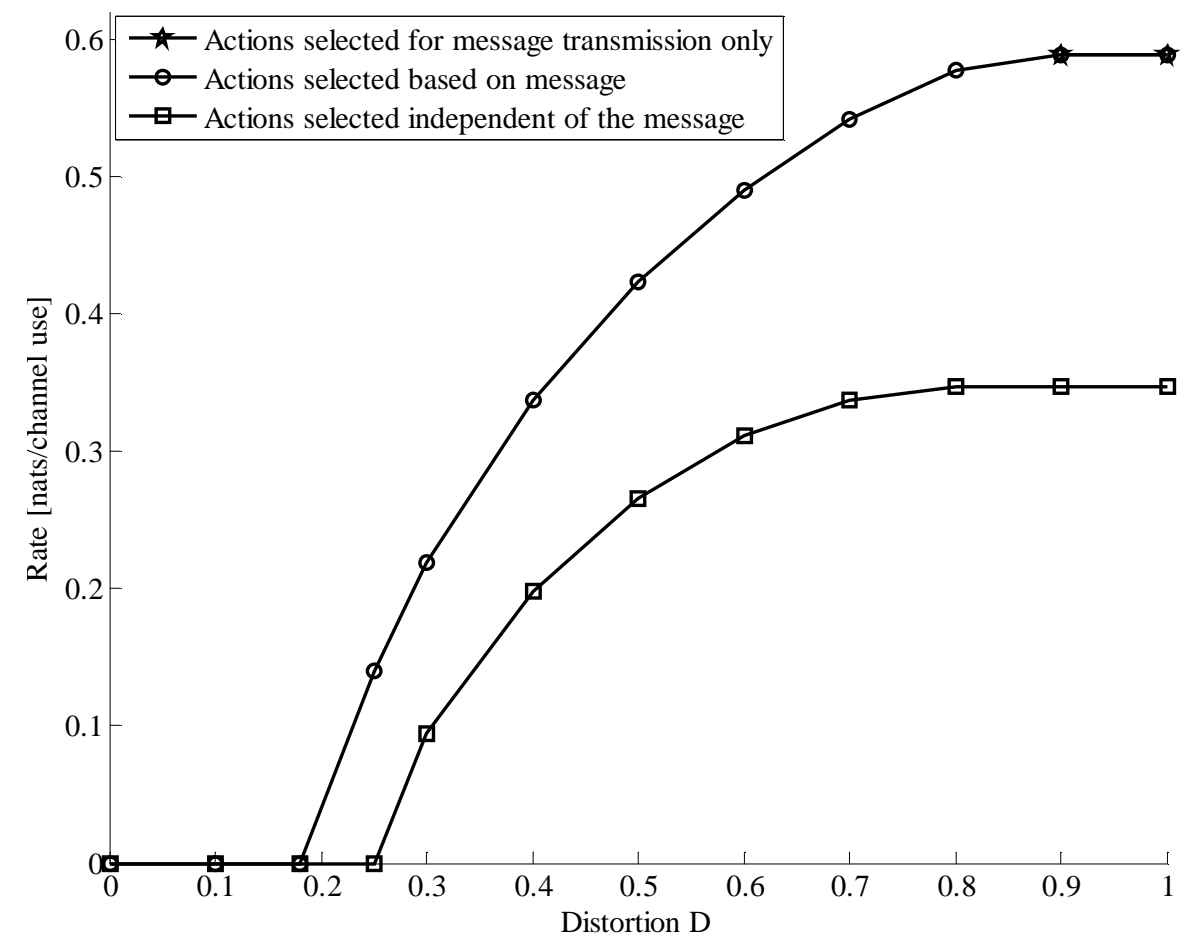

Figure 3. Achievable rates (constrained to a Gaussian joint distribution, see (16)) for the Gaussian model (14)-(15) versus distortion $D$ for $P_{A}=P_{X}=\sigma_{W}^{2}=\sigma_{Z}^{2}=1$.

$\prod_{i=1}^{n} p\left(y_{1 i}, y_{2 i} \mid x_{i}, s_{i}, a_{i}\right)$. The decoders, based on the received signals $Y_{1}^{n}$ and $Y_{2}^{n}$, estimate the messages $M_{1}$ and $M_{2}$, respectively.

Definition 4. An $\left(n, R_{1}, R_{2}, \Gamma, \epsilon\right)$ code for the model in Fig. 2 consists of an action encoder

$$
g_{1}: \mathcal{M}_{1} \times \mathcal{M}_{2} \rightarrow \mathcal{A}^{n}
$$

which maps messages $M_{1}$ and $M_{2}$ into an action sequence $A^{n}$; a sequence of channel encoders

$$
g_{2 i}: \mathcal{M}_{1} \times \mathcal{M}_{2} \times \mathcal{S}^{i} \rightarrow \mathcal{X}
$$

for $i \in[1, n]$ which map messages $M_{1}$ and $M_{2}$ and the first $i$ samples of the state sequence $S^{i}$ into the $i$ th symbol $X_{i}$; two decoding functions,

$$
h_{1}: \mathcal{Y}_{1}^{n} \rightarrow \mathcal{M}_{1}
$$

and $h_{2}: \mathcal{Y}_{2}^{n} \rightarrow \mathcal{M}_{2}$, 
which map the received sequences $Y_{1}^{n}$ and $Y_{2}^{n}$ into the estimated messages $\hat{M}_{1}$ and $\hat{M}_{2}$, respectively; such that the probability of error in decoding the messages $M_{1}$ and $M_{2}$ is small

$$
\operatorname{Pr}\left[\hat{M}_{j} \neq M_{j}\right] \leq \epsilon \text { for } j=1,2,
$$

and the cost constraint (8) is satisfied.

We note that, given the definitions above, the distribution of the random variables $\left(M_{1}, M_{2}, A^{n}\right.$, $\left.S^{n}, X^{n}, Y_{1}^{n}, Y_{2}^{n}\right)$ factorizes as

$$
\begin{aligned}
p\left(m_{1}, m_{2}, a^{n}, s^{n}, x^{n}, y_{1}^{n}, y_{2}^{n}\right)= & \frac{1}{2^{n\left(R_{1}+R_{2}\right)}} \delta\left[a^{n}-g_{1}\left(m_{1}, m_{2}\right)\right]\left\{\prod_{i=1}^{n} p\left(s_{i} \mid a_{i}\right)\right\} \\
& \cdot\left\{\prod_{i=1}^{n} \delta\left[x_{i}-g_{2 i}\left(m_{1}, m_{2}, s^{i}\right)\right] p\left(y_{1 i}, y_{2 i} \mid x_{i}, s_{i}, a_{i}\right)\right\},
\end{aligned}
$$

where the arguments of the pmf range in the alphabets of the corresponding random variables.

Given a cost $\Gamma$, a rate pair $\left(R_{1}, R_{2}\right)$ is said to be achievable if, for any $\epsilon>0$ and sufficiently large $n$, there a exists a $\left(n, R_{1}, R_{2}, \Gamma, \epsilon\right)$ code. The capacity region $\mathcal{C}(\Gamma)$ is defined as the closure of all rate pairs $\left(R_{1}, R_{2}\right)$ that are achievable given the cost $\Gamma$.

\section{B. Capacity-Cost Region}

In this section, a single-letter characterization of the capacity region is derived for the special case in which the channel is physically degraded in the sense that we have the condition

$$
p\left(y_{1}, y_{2} \mid x, s, a\right)=p\left(y_{1} \mid x, s, a\right) p\left(y_{2} \mid y_{1}\right),
$$

or equivalently we have that the Markov chain $\left(X_{i}, S_{i}, A_{i}\right)-Y_{1 i}-Y_{2 i}$ holds for all $i \in[1, n]$.

Proposition 5. The capacity region of the system in Fig. 2 under the degradedness condition (24) is given by the union of the rate pairs $\left(R_{1}, R_{2}\right)$ satisfying

$$
\begin{aligned}
R_{1} & \leq I\left(U_{1} ; Y_{1} \mid U_{2}\right) \\
\text { and } R_{2} & \leq I\left(U_{2} ; Y_{2}\right),
\end{aligned}
$$

where the mutual informations are evaluated with respect to the joint pmf $p\left(a, u_{1}, u_{2}, s, x, y_{1}, y_{2}\right)=p\left(u_{1}, u_{2}\right) \delta\left[a-f_{a}\left(u_{1}, u_{2}\right)\right] p(s \mid a) \delta\left[x-f_{x}\left(u_{1}, u_{2}, s\right)\right] p\left(y_{1} \mid x, s, a\right) p\left(y_{2} \mid y_{1}\right)$, 
for some pmfs $p\left(u_{1}, u_{2}\right)$ and deterministic functions $f_{a}: \mathcal{U}_{1} \times \mathcal{U}_{2} \rightarrow \mathcal{A}$ and $f_{x}: \mathcal{U}_{1} \times \mathcal{U}_{2} \times \mathcal{S} \rightarrow \mathcal{X}$ such that the inequality $\mathrm{E}[\gamma(A, X)] \leq \Gamma$ is satisfied. Auxiliary random variables $U_{1}$ and $U_{2}$ have finite alphabets.

The proof of achievability can be sketched as follows. The codewords $u_{2}^{n}\left(m_{2}\right)$, encoding message $m_{2} \in\left[1,2^{n R_{2}}\right]$, are generated independently and i.i.d. according to the pmf $p\left(u_{2}\right)$. Then, superimposed on each codeword $u_{2}^{n}\left(m_{2}\right), 2^{n R_{1}}$ codewords $u_{1}^{n}\left(m_{1}, m_{2}\right)$ are generated independently according to the distribution $\prod_{i=1}^{n} p\left(u_{1 i} \mid u_{2 i}\left(m_{2}\right)\right)$. To encode messages $\left(M_{1}, M_{2}\right)$, the action sequence $A^{n}$ is obtained as a deterministic function of $u_{1 i}\left(M_{1}, M_{2}\right)$ and $u_{2 i}\left(M_{2}\right)$ such that $A_{i}=f_{a}\left(u_{1 i}\left(M_{1}, M_{2}\right), u_{2 i}\left(M_{2}\right)\right)$ for all $i \in[1, n]$. The transmitted symbol $X_{i}$ is obtained instead as a function of $u_{1 i}\left(M_{1}, M_{2}\right), u_{2 i}\left(M_{2}\right)$, and of the $i$ th state symbol $S_{i}$ as $X_{i}=f_{x}\left(u_{1 i}\left(M_{1}, M_{2}\right), u_{2 i}\left(M_{2}\right), S_{i}\right)$. Decoder 2 decodes the codeword $u_{2}^{n}\left(m_{2}\right)$, while decoder 1 decodes both codewords $u_{2}^{n}\left(m_{2}\right)$ and $u_{1}^{n}\left(m_{1}, m_{2}\right)$. Using standard arguments, the rates (25) are easily shown to be achievable. The proof of the converse can be found in Appendix B.

Remark 6. If we let $p(s \mid a)=p(s)$ so that the channel is not action-dependent, Proposition 6 recovers Proposition 4 of [9] (see also [10]).

\section{A Binary Example}

In this section, we consider a special case of the model in Fig. 2 in which the action channel $p(s \mid a)$ is binary and given by

$$
S=A \oplus B,
$$

where the action $A$ is binary, $B \sim \operatorname{Ber}(b)$ and the transmission channels are given by

$$
\begin{aligned}
Y_{1} & =X \oplus S \oplus Z_{1}, \\
\text { and } Y_{2} & =Y_{1} \oplus \widetilde{Z}_{2},
\end{aligned}
$$

where $Z_{1} \sim \operatorname{Ber}\left(N_{1}\right)$ and $\widetilde{Z}_{2} \sim \operatorname{Ber}\left(\widetilde{N}_{2}\right)$ are independent of each other and of $B$. We select the cost metric as $\gamma(a, x)=x$. We define $N_{2}=N_{1} * \widetilde{N}_{2}=N_{1}\left(1-\widetilde{N}_{2}\right)+\widetilde{N}_{2}\left(1-N_{1}\right)$.

As a first remark, consider the ideal system with $b=0$ (i.e., no interference) and no cost constraint (i.e., $\Gamma=1 / 2$ ). The system reduces to a standard physical degraded binary symmetric 
broadcast channel, and thus the capacity region is given by the union over $\alpha \in[0,0.5]$ of the rate pairs satisfying the inequalities [12, p. 115]

$$
\begin{aligned}
R_{1} & \leq H\left(\alpha * N_{1}\right)-H\left(N_{1}\right) \\
\text { and } R_{2} & \leq 1-H\left(\alpha * N_{2}\right) .
\end{aligned}
$$

We observe that, by construction, this rate region sets an outer bound on the rate achievable in the system at hand. The outer bound above is in fact achievable by setting $X=B, U_{2} \sim \operatorname{Ber}(1 / 2)$, $U_{1}=U_{2} \oplus \widetilde{U}_{1}$ with $\widetilde{U}_{1} \sim \operatorname{Ber}(\alpha)$, and $A=U_{1}$ in (25), where $U_{2}$ and $\widetilde{U}_{1}$ are independent. This entails that, by leveraging the actions, the interference-free capacity region (29) is obtained for all cost constraints $\Gamma \geq b$. It can be instead seen that, if one is forced to set $A$ to be constant, achieving the rate region (29) requires a cost $\Gamma=1 / 2$, since $X$ needs to be distributed $\operatorname{Ber}(1 / 2)$. This example illustrates the advantage of being able to affect the state via actions selected as a function of the messages.

\section{Probing Capacity of Degraded Broadcast Channels}

In this section, we apply the setting of probing capacity introduced in [2] to the degraded broadcast channel. Following [2], the state sequence $S^{n}$ is thus assumed to be generated i.i.d. according to a pmf $p(s)$. Moreover, based on the messages $\left(M_{1}, M_{2}\right)$, the encoder selects an action sequence as in (18). However, here, through the choice of actions, the encoder affects the state information available at the encoder and the decoders, and not the state sequence $S^{n}$. Specifically, the encoder obtains partial state information $S_{e, i}=b_{e}\left(S_{i}, A_{i}\right)$, and the decoders obtain partial state informations $S_{d_{1, i}}=b_{d_{1}}\left(S_{i}, A_{i}\right)$ and $S_{d_{2, i}}=b_{d_{2}}\left(S_{d_{1, i}}\right)$, respectively, where $i \in[1, n]$, and $b_{e}: \mathcal{S} \times \mathcal{A} \rightarrow \mathcal{S}_{e}, b_{d_{1}}: \mathcal{S} \times \mathcal{A} \rightarrow \mathcal{S}_{d_{1}}$ and $b_{d_{2}}: \mathcal{S}_{d_{1}} \rightarrow \mathcal{S}_{d_{2}}$ are deterministic functions for given alphabets $\mathcal{S}_{e}, \mathcal{S}_{d_{1}}$ and $\mathcal{S}_{d_{2}}$. Note that the state information available at decoder 2 is degraded with respect to that of decoder 1 (i.e., it is a function of the latter). As in [2], we assume that the state information at the encoder is characterized as

$$
S_{e, i}=b_{e}\left(S_{i}, A_{i}\right)=\left\{\begin{array}{ll}
S_{i} & \text { if } A_{i}=1 \\
* & \text { if } A_{i}=0
\end{array},\right.
$$

where $*$ represents the absence of channel state information at the encoder. Moreover, the state information $S_{e, i}$ is assumed to be available causally at the encoder so that the encoding function 
is $g_{2 i}: \mathcal{M}_{1} \times \mathcal{M}_{2} \times \mathcal{S}_{e}^{i} \rightarrow \mathcal{X}$ (cf. (19)). The rest of the code definition is similar to Definition 4 with the caveat that the decoder 1 and 2 have available also the information sequences $S_{d_{1}}^{n}$ and $S_{d_{2}}^{n}$, respectively.

We note that, given the definitions above, the distribution of the random variables $\left(M_{1}, M_{2}, A^{n}\right.$, $\left.S^{n}, X^{n}, Y_{1}^{n}, Y_{2}^{n}\right)$ factorizes as

$$
\begin{array}{r}
p\left(m_{1}, m_{2}, a^{n}, s^{n}, s_{e}^{n}, s_{d_{1}}^{n}, s_{d_{2}}^{n}, x^{n}, y_{1}^{n}, y_{2}^{n}\right)=\frac{1}{2^{n\left(R_{1}+R_{2}\right)}} \delta\left[a^{n}-g_{1}\left(m_{1}, m_{2}\right)\right] \\
\cdot\left\{\prod_{i=1}^{n} p\left(s_{i}\right) \delta\left[s_{e, i}-b_{e}\left(s_{i}, a_{i}\right)\right] \delta\left[s_{d_{1, i}}-b_{d_{1}}\left(s_{i}, a_{i}\right)\right] \delta\left[x_{i}-g_{2}\left(m_{1}, m_{2}, s_{e}^{i}\right)\right\}\right. \\
\cdot\left\{\prod_{i=1}^{n} \delta\left[s_{d_{2, i}}-b_{d_{2}}\left(s_{d_{1, i}}\right)\right] p\left(y_{1 i}, y_{2 i} \mid x_{i}, s_{i}, a_{i}\right)\right\}
\end{array}
$$

where the arguments of the pmf range in the alphabets of the corresponding random variables.

As discussed below, the setting at hand, which we refer to as having a probing encoder, is a special case of the one studied in Sec. II-A Therefore, we can leverage Proposition 5 to obtain the following result.

Proposition 7. The capacity region of the system in Fig. 2 under the degradedness condition (24) and with a probing encoder is given by the union of the rate pairs $\left(R_{1}, R_{2}\right)$ satisfying

$$
\begin{aligned}
R_{1} & \leq I\left(U_{1} ; Y_{1}, S_{d_{1}} \mid U_{2}\right) \\
\text { and } R_{2} & \leq I\left(U_{2} ; Y_{2}, S_{d_{2}}\right)
\end{aligned}
$$

where the mutual informations are evaluated with respect to the joint pmf

$$
\begin{aligned}
p\left(a, u_{1}, u_{2}, s, s_{e}, s_{d_{1}}, s_{d_{2}}, x, y_{1}, y_{2}\right)= & p(s) p\left(u_{1}, u_{2}\right) \delta\left[a-f_{a}\left(u_{1}, u_{2}\right)\right] \delta\left[s_{e}-b_{e}(s, a)\right] \\
& \cdot \delta\left[s_{d_{1}}-b_{d_{1}}(s, a)\right] \delta\left[s_{d_{2}}-b_{d_{2}}\left(s_{d_{1}}\right)\right] \\
& \cdot \delta\left[x-f_{x}\left(u_{1}, u_{2}, s_{e}\right)\right] p\left(y_{1} \mid x, s, a\right) p\left(y_{2} \mid y_{1}\right),
\end{aligned}
$$

for some pmf $p\left(u_{1}, u_{2}\right)$ and deterministic functions $f_{a}: \mathcal{U}_{1} \times \mathcal{U}_{2} \rightarrow \mathcal{A}$ and $f_{x}: \mathcal{U}_{1} \times \mathcal{U}_{2} \times \mathcal{S}_{e} \rightarrow \mathcal{X}$ such that the inequality $\mathrm{E}[\gamma(A, X)] \leq \Gamma$ is satisfied.

Proof: The result is obtained by noticing that the setting described above is a special case 
of the one described in Sec. 【II-A by making the following substitutions

$$
\begin{aligned}
S & \rightarrow S_{e} \\
\text { and } Y_{j} & \rightarrow\left(Y_{j}, S_{d_{j}}\right) \text { for } j=1,2 .
\end{aligned}
$$

To see this, we show that the pmf (31) reduces to (23) under the given substitutions. Specifically, by marginalizing (31) over $S^{n}$ we have

$$
\begin{array}{r}
\frac{1}{2^{n\left(R_{1}+R_{2}\right)}} \delta\left[a^{n}-g_{1}\left(m_{1}, m_{2}\right)\right]\left\{\prod_{i=1}^{n} \delta\left[x_{i}-g_{2}\left(m_{1}, m_{2}, s_{e}^{i}\right)\right] \delta\left[s_{d_{2, i}}-b_{d_{2}}\left(s_{d_{1, i}}\right)\right] p\left(y_{2 i} \mid y_{1 i}\right)\right\} \\
\prod_{i=1}^{n} \sum_{s_{i} \in \mathcal{S}}\left\{p\left(s_{i}\right) \delta\left[s_{e, i}-b_{e}\left(s_{i}, a_{i}\right)\right] \delta\left[s_{d_{1, i}}-b_{d_{1}}\left(s_{i}, a_{i}\right)\right] p\left(y_{1 i} \mid x_{i}, s_{i}, a_{i}\right)\right\} .
\end{array}
$$

The terms outside the summation in (35) are equal to the corresponding terms in (23) under the substitutions (34). For the remaining terms, we observe that, for $a_{i}=0$, we have $S_{e, i}=*$, and thus $p\left(s_{e, i} \mid a_{i}\right)=\delta\left[s_{e, i}-*\right]$ and

$$
p\left(y_{1 i}, s_{d_{1 i}} \mid x_{i}, s_{e, i}, a_{i}\right)=\sum_{s_{i} \in \mathcal{S}} p\left(s_{i}\right) \delta\left[s_{d_{1, i}}-b_{d_{1}}\left(s_{i}, a_{i}\right)\right] p\left(y_{1 i} \mid x_{i}, s_{i}, a_{i}\right) ;
$$

instead, for $a_{i}=1$ we have $S_{e, i}=S_{i}$, and thus $p\left(s_{e, i} \mid a_{i}\right)=\operatorname{Pr}\left[S_{i}=s_{e, i}\right]$ and

$$
p\left(y_{1 i}, s_{d_{1 i}} \mid x_{i}, s_{e, i}, a_{i}\right)=\delta\left[s_{d_{1, i}}-b_{d_{1}}\left(s_{e, i}, a_{i}\right)\right] p\left(y_{1 i} \mid x_{i}, s_{e, i}, a_{i}\right)
$$

which completes the proof.

\section{CONCLUDING REMARKS}

Action-dependent channels are useful abstractions of two-phase communication scenarios. This paper has reported on two variations on this theme, namely the problem of message and state transmission in an action-dependent channel and the degraded action-dependent broadcast channel. Under given assumptions, we have characterized the information-theoretic performance of these systems. The analytical results, and specific examples, emphasize the importance of jointly designing the transmission strategy across the two communication phases. 


\section{ApPendix A: Proof of Proposition 2}

We first observe that given the probability of error constraint (6) we have the Fano inequality

$$
H\left(M \mid Y^{n}\right) \leq n \delta(\epsilon)
$$

where the notation $\delta(\epsilon)$ represents any function such that $\delta(\epsilon) \rightarrow 0$ as $\epsilon \rightarrow 0$, and that given the CR constraint (9), we have the Fano inequality

$$
H\left(\psi \mid Y^{n}\right) \leq n \delta(\epsilon)
$$

We can then write

$$
\begin{aligned}
& n R=H(M) \stackrel{(a)}{\leq} I\left(M ; Y^{n}\right)+n \delta(\epsilon) \\
& \stackrel{(b)}{=} I\left(M ; Y^{n}\right)-I\left(M ; S^{n} \mid A^{n}\right)+n \delta(\epsilon) \\
& =I\left(\psi, M ; Y^{n}\right)-I\left(\psi ; Y^{n} \mid M\right)-I\left(\psi M ; S^{n} \mid A^{n}\right)+I\left(\psi ; S^{n} \mid A^{n}, M\right)+n \delta(\epsilon) \\
& =I\left(\psi, M ; Y^{n}\right)-H(\psi \mid M)+H\left(\psi \mid M, Y^{n}\right)-I\left(\psi, M ; S^{n} \mid A^{n}\right)+H\left(\psi \mid A^{n}, M\right) \\
& -H\left(\psi \mid A^{n}, M, S^{n}\right)+n \delta(\epsilon) \\
& =I\left(\psi, M ; Y^{n}\right)-I\left(\psi ; A^{n} \mid M\right)+H\left(\psi \mid M, Y^{n}\right)-I\left(\psi, M ; S^{n} \mid A^{n}\right) \\
& -H\left(\psi \mid A^{n}, M, S^{n}\right)+n \delta(\epsilon) \\
& \stackrel{(c)}{\leq} I\left(\psi, M ; Y^{n}\right)-I\left(\psi, M ; S^{n} \mid A^{n}\right)+n \delta(\epsilon) \\
& =\sum_{i=1}^{n} I\left(\psi, M ; Y_{i} \mid Y^{i-1}\right)-I\left(\psi, M ; S_{i} \mid S_{i+1}^{n}, A^{n}\right)+n \delta(\epsilon) \\
& \stackrel{(d)}{\leq} \sum_{i=1}^{n} H\left(Y_{i}\right)-H\left(Y_{i} \mid Y^{i-1}, \psi, M, S_{i+1}^{n}, A^{n}\right)-H\left(S_{i} \mid S_{i+1}^{n}, A^{n}\right) \\
& +H\left(S_{i} \mid Y^{i-1}, \psi, M, S_{i+1}^{n}, A^{n}\right)+n \delta(\epsilon) \\
& \stackrel{(e)}{=} \sum_{i=1}^{n} H\left(Y_{i}\right)-H\left(Y_{i} \mid U_{i}\right)-H\left(S_{i} \mid A_{i}\right)+H\left(S_{i} \mid U_{i}, A_{i}\right)+n \delta(\epsilon) \\
& =\sum_{i=1}^{n} I\left(U_{i} ; Y_{i}\right)-I\left(U_{i} ; S_{i} \mid A_{i}\right)+n \delta(\epsilon)
\end{aligned}
$$

where (a) follows due to Fano's inequality as in (36); (b) follows using the Markov chain $M-A^{n}-S^{n} ;(c)$ follows by (37a) and since mutual information is non-negative (recall that by definition $2 n \delta(\epsilon)=n \delta(\epsilon)$ ); (d) follows using the same steps provided in the proof of 
Theorem 1 in [1, eq. (9)-(12)] by substituting $M$ with $(M, \psi)$; and (e) follows by defining $U_{i} \triangleq\left(Y^{i-1}, \psi, M, S_{i+1}^{n}, A^{n \backslash i}\right)$ and because we have the Markov relation $S_{i}-A_{i}-\left(S_{i+1}^{n}, A^{n \backslash i}\right)$.

Defining $Q$ to be a random variable uniformly distributed over $[1, n]$ and independent of $\left(A^{n}, S^{n}, U^{n}, X^{n}, Y^{n}\right)$, and with $A \triangleq A_{Q}, S \triangleq S_{Q}, X \triangleq X_{Q}, Y \triangleq Y_{Q}$ and $U \triangleq\left(U_{Q}, Q\right)$, from (47) we have

$$
\begin{aligned}
R & \leq I(U ; Y \mid Q)-I(U ; S \mid A, Q)+\delta(\epsilon) \\
& \stackrel{(a)}{=} H(Y \mid Q)-H(Y \mid U)-H(S \mid A, Q)+H(S \mid A, U)+\delta(\epsilon) \\
& \stackrel{(b)}{\leq} H(Y)-H(Y \mid U)-H(S \mid A)+H(S \mid A, U)+\delta(\epsilon) \\
& =I(U ; Y)-I(U ; S \mid A)+\delta(\epsilon)
\end{aligned}
$$

where (a) follows using the definition of $U$ and (b) follows because conditioning reduces entropy. Moreover, from (8), we have

$$
\Gamma+\epsilon \geq \frac{1}{n} \sum_{i=1}^{n} \mathrm{E}\left[\gamma\left(A_{i}, X_{i}\right)\right]=\mathrm{E}[\gamma(A, X)] .
$$

Next, define $\hat{S}_{i}=\psi_{i}\left(S^{n}\right)$ and $\hat{S}=\hat{S}_{Q}$, where $\psi_{i}\left(S^{n}\right)$ represents the $i$ th symbol of $\psi\left(S^{n}\right)$. Moreover, let $\mathcal{B}$ be the event $\mathcal{B}=\left\{\psi\left(S^{n}\right) \neq h_{2}\left(Y^{n}\right)\right\}$. Using the $\mathrm{CR}$ requirement (9), we have $\operatorname{Pr}(\mathcal{B}) \leq \epsilon$. We can then calculate the distortion as (we drop the dependence of $\mathrm{h}_{2 i}$ on $Y^{n}$ for simplicity of notation)

$$
\begin{aligned}
\mathrm{E}[d(S, \hat{S})]=\frac{1}{n} \sum_{i=1}^{n} \mathrm{E}\left[d\left(S_{i}, \hat{S}_{i}\right)\right] & =\frac{1}{n} \sum_{i=1}^{n} \mathrm{E}\left[d\left(S_{i}, \hat{S}_{i}\right) \mid \mathcal{B}\right] \operatorname{Pr}(\mathcal{B})+\frac{1}{n} \sum_{i=1}^{n} \mathrm{E}\left[d\left(S_{i}, \hat{S}_{i}\right) \mid \mathcal{B}^{c}\right] \operatorname{Pr}\left(\mathcal{B}^{c}\right) \\
& \stackrel{(a)}{\leq} \frac{1}{n} \sum_{i=1}^{n} \mathrm{E}\left[d\left(S_{i}, \hat{S}_{i}\right) \mid \mathcal{B}^{c}\right] \operatorname{Pr}\left(\mathcal{B}^{c}\right)+\epsilon D_{\max } \\
& \stackrel{(b)}{\leq} \frac{1}{n} \sum_{i=1}^{n} \mathrm{E}\left[d\left(S_{i}, h_{2 i}\right)\right]+\epsilon D_{\max } \\
& \stackrel{(c)}{\leq} D+\epsilon D_{\max },
\end{aligned}
$$

where (a) follows using the fact that $\operatorname{Pr}(\mathcal{B}) \leq \epsilon$ and that the distortion is upper bounded by $D_{\max } ;(b)$ follows by the definition of $\hat{S}_{i}$ and $\mathcal{B}$; and (c) follows by (7).

To bound the cardinality of auxiliary random variable $U$, we first observe that the distribution of the variables $(A, U, S, X, Y, \hat{S})$ identified above factorizes as

$$
p(a, u, s, x, y)=p(u) p(a, s, x \mid u) p(y \mid x, s, a),
$$


and $\hat{S}$ is a deterministic function $\phi(U)$. Therefore, for fixed $p(y \mid x, s, a)$, the characterization in Proposition 2 can be expressed in terms of integrals $\int g_{j}(p(a, s, x \mid u)) d F(u)$ for $j=1, \ldots,|\mathcal{A}| \times$ $|\mathcal{S}| \times|\mathcal{X}|+2$, of functions $g_{j}($.$) that are continuous over pmf on the alphabet |\mathcal{A}| \times|\mathcal{S}| \times|\mathcal{X}|$. Specifically, we have $g_{j}$ for $j=1, \ldots,|\mathcal{A}| \times|\mathcal{S}| \times|\mathcal{X}|-1$ given by $p(a, s, x)$ for all values of $a \in \mathcal{A}$, $s \in \mathcal{S}$, and $x \in \mathcal{X}$ (except one); $g_{|\mathcal{A}| \times|\mathcal{S}| \times|\mathcal{X}|}=H(Y \mid U=u) ; g_{|\mathcal{A}| \times|\mathcal{S}| \times|\mathcal{X}|+2}=H(S \mid A, U=u) ;$ and $g_{|\mathcal{A}| \times|\mathcal{S}| \times|\mathcal{X}|+1}=\mathrm{E}[d(S, \hat{S}) \mid U=u]$. The cardinality bound follows by invoking FenchelEggleston-Caratheodory Theorem [12, Appendix C]. We finally observe that the joint distribution (51) can be written as (12) without loss of generality, since $U$ can always contain $A$ without reducing rate (11).

\section{APPENDiX B: Proof OF PROPOSITION 5}

The proof is similar to that given in [9] (see also [10]), although care must be taken to properly account for the presence of the actions. We first observe that given the probability of error constraint (22), we have the Fano inequality $H\left(M_{j} \mid Y_{j}^{n}\right) \leq n \delta(\epsilon)$ for $j=1,2$. We can then write the inequalities

$$
\begin{aligned}
n R_{2} & =H\left(M_{2}\right) \stackrel{(a)}{\leq} I\left(M_{2} ; Y_{2}^{n}\right)+n \delta(\epsilon) \\
& \stackrel{(b)}{=} \sum_{i=1}^{n} I\left(M_{2} ; Y_{2 i} \mid Y_{2}^{i-1}\right)+n \delta(\epsilon) \\
& \stackrel{(c)}{\leq} \sum_{i=1}^{n} I\left(M_{2}, Y_{2}^{i-1} ; Y_{2 i}\right)+n \delta(\epsilon) \\
& \stackrel{(d)}{\leq} \sum_{i=1}^{n} I\left(M_{2}, Y_{2}^{i-1}, Y_{1}^{i-1} ; Y_{2 i}\right)+n \delta(\epsilon) \\
& \stackrel{(e)}{=} \sum_{i=1}^{n} I\left(U_{2 i} ; Y_{2 i}\right)+n \delta(\epsilon),
\end{aligned}
$$

where (a) follows due to Fano's inequality as in (36); (b) follows by using the chain rule for mutual information; $(c)$ and $(d)$ follow because conditioning increases entropy; and $(e)$ follows by defining $U_{2 i} \triangleq\left(M_{2}, Y_{1}^{i-1}\right)$ and noting the Markov relation $Y_{2}^{i-1}-\left(Y_{1}^{i-1}, M_{2}\right)-Y_{2 i}$ due to 
the degradedness property (24). We also have the inequalities

$$
\begin{aligned}
n R_{1} & =H\left(M_{1}\right) \stackrel{(a)}{\leq} I\left(M_{1} ; Y_{1}^{n}\right)+n \delta(\epsilon) \\
& \stackrel{(b)}{\leq} I\left(M_{1} ; Y_{1}^{n} \mid M_{2}\right)+n \delta(\epsilon) \\
& \stackrel{(c)}{=} \sum_{i=1}^{n} I\left(M_{1} ; Y_{1 i} \mid Y_{1}^{i-1}, M_{2}\right)+n \delta(\epsilon) \\
& \stackrel{(d)}{\leq} \sum_{i=1}^{n} I\left(M_{1}, Y_{1}^{i-1}, S^{i-1} ; Y_{1 i} \mid Y_{1}^{i-1}, M_{2}\right)+n \delta(\epsilon) \\
& \stackrel{(d)}{\leq} \sum_{i=1}^{n} I\left(U_{1 i} ; Y_{1 i} \mid U_{2 i}\right)+n \delta(\epsilon),
\end{aligned}
$$

where (a) follows due to Fano's inequality as in (36); (b) follows because $M_{1}$ and $M_{2}$ are independent and since conditioning reduces entropy; $(c)$ follows using the chain rule for mutual information; $(d)$ follows since conditioning decreases entropy; and (e) follows by defining $U_{1 i} \triangleq$ $\left(M_{1}, Y_{1}^{i-1}, S^{i-1}\right)$. Let $Q$ be a random variable uniformly distributed over $[1, n]$ and independent of $\left(A^{n}, S^{n}, U_{1}^{n}, U_{2}^{n}, X^{n}, Y_{1}^{n}, Y_{2}^{n}\right)$ and define $A \triangleq A_{Q}, S \triangleq S_{Q}, X \triangleq X_{Q}, Y_{1} \triangleq Y_{1 Q}, Y_{2} \triangleq Y_{2 Q}$, $U_{1} \triangleq\left(U_{1 Q}, Q\right)$, and $U_{2} \triangleq\left(U_{2 Q}, Q\right)$. We easily see that, with these definitions, the sum (56) is upper bounded by $I\left(U_{2} ; Y_{2}\right)$, and (61) equals $I\left(U_{1} ; Y_{1} \mid U_{2}\right)$. Moreover, note that, from the definitions above, $X$ is a function of $U_{1}, U_{2}$ and $S$, given the encoding function (19). Similarly, $A$ is a function of $\left(U_{1}, U_{2}\right)$ given (18). We also have the Markov relationship $\left(U_{1}, U_{2}\right)-A-S$ as it can be easily checked by using the d-separation principle [13]. Finally, from (8), we have

$$
\Gamma+\epsilon \geq \frac{1}{n} \sum_{i=1}^{n} \mathrm{E}\left[\gamma\left(A_{i}, X_{i}\right)\right]=\mathrm{E}[\gamma(A, X)]
$$

which completes the proof.

\section{ACKNOWLEDGMENT}

The work of O. Simeone is supported by the U.S. National Science Foundation under grant CCF-0914899.

\section{REFERENCES}

[1] T. Weissman, "Capacity of channels with action-dependent states," IEEE Trans. Inform. Theory, vol. 56, no. 11, pp. 5396-5411, Nov. 2010.

[2] H. Asnani, H. Permuter, and T. Weissman, "Probing capacity," IEEE Trans. Inform. Theory, vol. 57, no. 11, pp. 7317-7332, Nov. 2011. 
[3] K. Kittichokechai, T. J. Oechtering, and M. Skoglund, "Coding with action-dependent side information and additional reconstruction requirements," http://arxiv.org/abs/1202.1484, Feb 2012.

[4] C. Choudhuri and U. Mitra, "Action dependent strictly causal state communication," http://arxiv.org/abs/1202.0934, Feb. 2012.

[5] Y. Steinberg, "Coding and common reconstruction," IEEE Trans. Inform. Theory, vol. 55, no. 11, 2009.

[6] Y.-H. Kim, A. Sutivong, and T. M. Cover, "State amplification,” IEEE Trans. Inform. Theory, vol. 54, no. 5, pp. 1850-1859, May 2008.

[7] B. Ahmadi and O. Simeone, "On channels with action-dependent states," http://arxiv.org/abs/1202.4438v1 Feb. 2012.

[8] Y. Steinberg and T. Weissman, "The degraded broadcast channel with action-dependent states," in Proc. IEEE Symp. Inform. Theory, Boston, USA, Jul. 2012.

[9] Y. Steinberg, "Coding for the degraded broadcast channel with random parameters, with causal and noncausal side information," IEEE Trans. Inform. Theory, vol. 51, no. 8, pp. 2867-2877, Aug. 2005.

[10] S. Sigurjonsson and Y. H. Kim, "On multiple user channels with causal state information at the transmitters," in Proc. IEEE Symp. Inform. Theory, Adelaide, Australia, pp. 72-76, Sept. 2005.

[11] Y. Steinberg and S. Shamai, "Achievable rates for the broadcast channel with states known at the transmitter," in Proc. IEEE Symp. Inform. Theory, Adelaide, Australia, pp. 2184 - 2188 , Sept. 2005.

[12] A. El Gamal and Y. Kim, Network Information Theory, Cambridge University Press, Dec 2011.

[13] G. Kramer, Topics in Multi-User Information Theory, Now Publishers, 2008. 